\title{
An Algorithm to Determine the Extent of an Epidemic Spread: A NetLogo Modeling Approach
}

\author{
Jerry John Kponyo ${ }^{1}$, Kenneth Coker ${ }^{1}$, Justice Owusu Agyemang ${ }^{1}$, Joyce Der ${ }^{2,3}$ \\ ${ }^{1}$ Department of Electrical Engineering, College of Engineering, Kwame Nkrumah University of Science and Technology, Kumasi, Ghana \\ ${ }^{2}$ School of Public Health, University of Health and Allied Sciences, Ho, Ghana \\ ${ }^{3}$ London School of Hygiene and Tropical Medicine, London, United Kingdom
}

Email address:

jjkponyo@ieee.org (J. J. Kponyo), kennethcoker25@gmail.com (K. Coker), justiceowusuagyemang@gmail.com (J. O. Agyemang), der.joyceb@gmail.com (J. Der)

\section{To cite this article:}

Jerry John Kponyo, Kenneth Coker, Justice Owusu Agyemang, Joyce Der. An Algorithm to Determine the Extent of an Epidemic Spread: A NetLogo Modeling Approach. Engineering and Applied Sciences. Vol. 4, No. 4, 2019, pp. 74-78. doi: 10.11648/j.eas.20190404.11

Received: January 11, 2019; Accepted: August 7, 2019; Published: August 23, 2019

\begin{abstract}
The outbreaks of infectious diseases have had a huge impact on the human society. Researchers have developed models aimed at understanding how various infectious diseases spread in communities and also proposed control measures that can minimize or stop the spread of the diseases. Most researchers have developed stochastic mathematical models which are used in predicting the occurrence of an epidemic. Most of the proposed models do not employ the use of system dynamics hence making it difficult to adopt the same model in predicting the behavior of other epidemic diseases. This research work focuses on the use of system dynamics in predicting the extent of an epidemic spread so that effective preventive and quarantine measures can be put in place to curb that epidemic. The SIR model forms the basis of the model. The model was developed in NetLogo. Disease parameters and environmental conditions play a role in the spread of an epidemic. Due to this the parameters used in the model included initial population, infectiousness, fatality rate, days to recover, hygiene, vaccination, travel-openings and the number of doctors within the community. The efficiency of the developed model was tested using data from two disease outbreaks: Ebola and Influenza. The model proved itself to be efficient in predicting the infected and death cases which were very close to the real-life data.
\end{abstract}

Keywords: NetLogo, SIR, Epidemic, Influenza, Ebola

\section{Introduction}

The outbreak of an infectious diseases tends to have a huge impact on the society all through history. In the $14^{\text {th }}$ century, the black plague killed between $30-50 \%$ of Europe's population. In the year 1720 , a plague epidemic decimated almost half of the population of Marseille and one-fourth of the population of Provence. In 1918-1919, the Spanish flu killed between 50-100 million humans (about 3-5\% of human population). Smallpox had existed for more than 3000 years, killing many millions of people, before it was eradicated after a long and intense vaccination effort lead by WHO. Recently, some of the diseases causing most harm (casualties, suffering and/or economically) to humans have been, HIV, influenza, foot and mouth disease, malaria and measles, and other diseases have caused unexpected dramatic outbreaks with high mortality rate (eg. SARS and Ebola) [1].

A paramount goal for public health is to increase understanding of how various infectious diseases spread within communities, with the goal of minimizing or even stopping the spread through various control measures (vaccination, quarantine, isolation etc). This has caused researchers to develop models that can help us understand how some of these infectious diseases behave in order for appropriate measures to be put in place to curb these diseases.

This paper presents a model that predicts the extent of an epidemic outbreak by providing a forecast on infected and death cases using a number of disease parameters. The rest of this paper is organized as follows: Section 2 reviews related literature. Section 3 presents the methodology. Section 4 presents the results of the study. Section 5 is the conclusion and recommendation. 


\section{Review of Related Works}

Many diseases have been found to be endemic and exhibit regular oscillatory levels of incidence in large population [2-5].

An algorithm based on the median ball principle was developed with the aim of predicting the infected and recovery rate of a virus that causes an epidemic using highly noisy and incomplete data [6].

A standard regression analysis based on the timing of the maximum prevalence of a pandemic wave, along with its amplitude and duration, has been used to develop an epidemic model that provides a real-time epidemic forecasting for pandemic influenza [7].

Serfling used regression analysis to derive epidemic thresholds from weekly pneumonia and influenza deaths for 108 US cities [8]. This method has been adapted to look at reported caseloads in France during the late 1980s [9], and fixed thresholds have been used to consider regional increases as a predictor of national epidemics [10].

A kriging method has also been applied to study spatial effects in France [11] as has the method of analogues [12]. Others have used time-series analysis based on previous epidemic data to define epidemic thresholds [13], or applied a semi-quantitative method based on a predefined epidemic threshold [14]. A linear regression model, applied to cumulative cases at the steepest ascent of the epidemic curve has also been used to predict weekly incidence [15].

Mills et al. [16] recently considered the transmission of the 1918 influenza pandemic and estimated an effective reproduction number of 2-3 by linearizing an SEIR model. Wallinga \& Tuenis [17] developed a real-time prediction tool for SARS which requires detailed epidemiological information and does not delete the susceptible population.

Manli et al. [18] proposed a stochastic SIR epidemic model with seasonal variation and saturated incidence. The researchers developed a mathematical model to obtain the threshold of the stochastic system which determines whether an epidemic occurs or not.

\section{Methodology}

The proposed model considers environmental factors and disease parameters which affect epidemic spread. The environmental conditions are affected by initial population, number of doctors in the community, travel openings, the hygienic condition of the community, the presence or absence of vaccination and whether there is medication or not. Disease parameters such as the infection rate that tells how fast the disease is contracted, the fatality rate that shows the rate at which the disease kills and the recovery rate were also included since $100 \%$ of those who come down with an epidemic do not die. Finally, the model also takes into consideration the days-to-recover from the epidemic.

The proposed algorithm is based on the SIR compartment model shown in Figure 1. The ' $\mathrm{S}$ ' indicates the Susceptible, that is those who are exposed and can be infected by the disease causing the epidemic, the 'I' indicates the Infected, that is those who have been infected by the disease and can spread it to the susceptible and the ' $R$ ' indicates the Recovered, that is those who have been treated and are immune to the epidemic. The SIR values are probability values so they sum up to 1 . Based on the SIR theory, individuals leave the susceptible group only to join the infected and individuals also leave the infected group only to join the recovered.

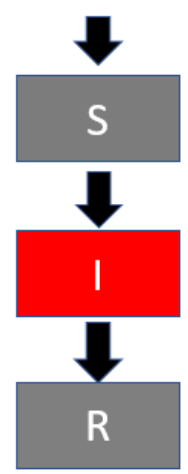

Susceptible:

can catch the disease

$$
\frac{\partial S}{\partial t}=-\beta S I \cdots(1)
$$

Infected:

have caught the disease and can spread it

to susceptible $\quad \frac{\partial I}{\partial t}=\beta S I-\gamma I \cdots(2)$

Recovered:

have recovered from the disease and are immune

$$
\begin{array}{r}
\frac{\partial R}{\partial t}=\gamma I \cdots(3) \\
\mathrm{S}+\mathrm{I}+\mathrm{R}=1
\end{array}
$$

Figure 1. The SIR compartment model.

The relationship between the SIR compartments is shown in Figure 2.

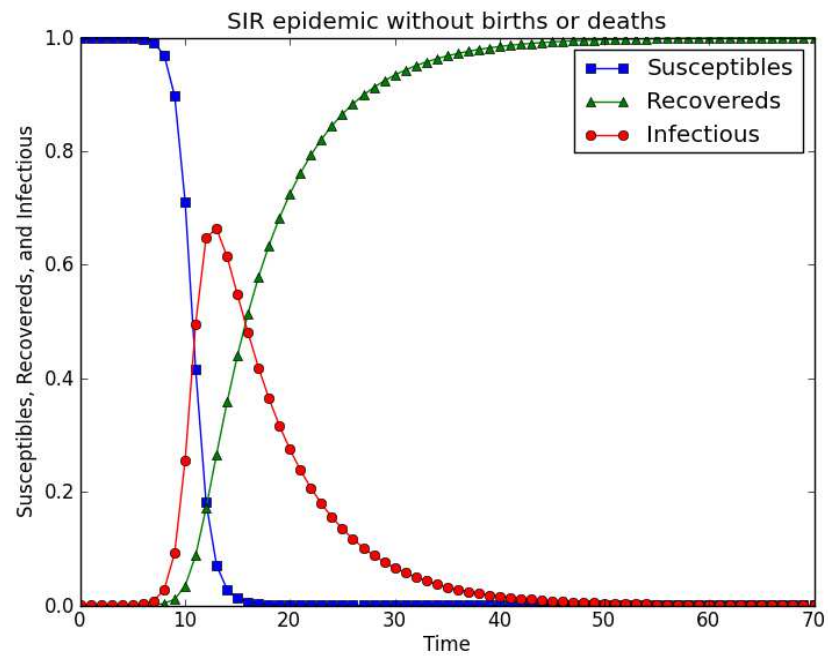

Figure 2. Relationship between the SIR compartments.

The graph shows clearly at the interception points that the various compartments are dependent on each other. The susceptible group begins with a probability of 1 and starts decreasing at a rate equal to the infection rate. The infected group also starts decreasing at a rate equal to the recovery rate and the decrease translates to an increase in the recovery compartment.

The entire modeling was done in NetLogo. The model was completed in three phases. There was an initial stage where a simple model was set up. Environmental and disease parameters were then included in the model to make it more realistic. Further work was done to include travel openings to mimic inter-epidemic spreads. The simulation was run till the 
last person in contact with the epidemic died. The use of sliders was employed to select parameters to suite a particular epidemic easier. The model is implemented in three phases and the user interface was made as user friendly as possible.

\subsection{Phase 1}

A simple dynamics model was developed where people catch the epidemic, spread it to others and die. This stage contained less data. It was used as a case study to project how the final model will look like. Figure 3 shows the NetLogo interface illustrating the infected and the susceptible.

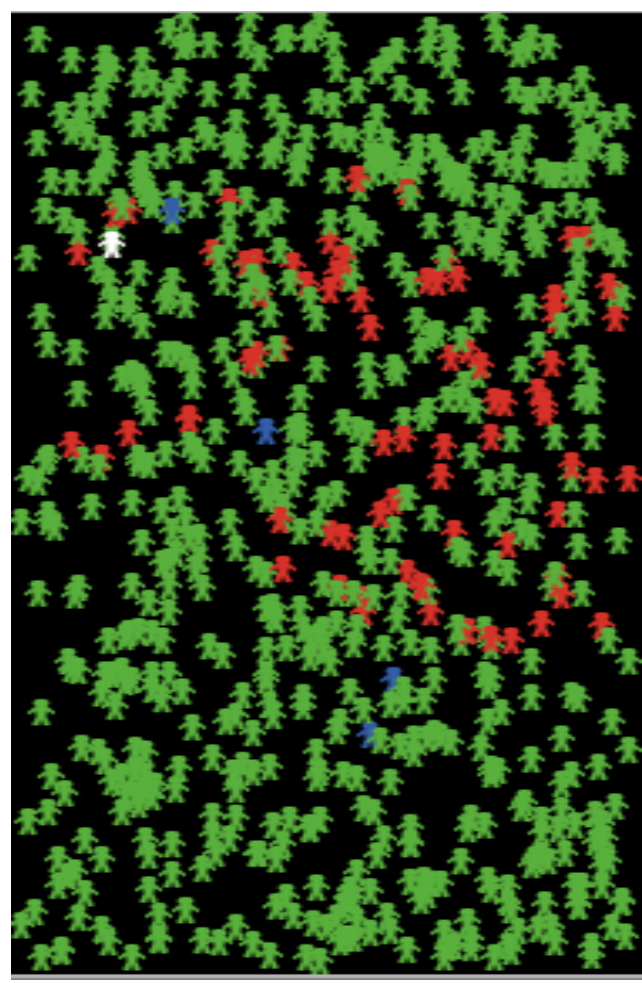

Figure 3. NetLogo interface for Phase 1.

NetLogo is an agent-based modeling tool and as such individuals that make up the population are represented as "turtles" in the model. The turtles in red represent those with the disease, the turtles in blue represent the healthy and the turtles in green are the susceptible and prone to catching the disease.

\subsection{Phase 2}

This was the stage of the model where there was an inclusion of environmental conditions and disease parameters to make it mimic real-life situations. The environmental conditions included were: initial population, number of doctors, travel openings, the hygienic conditions of the environment, medication and vaccination. The disease parameters included were: infection rate, fatality rate, recovery rate and the days-to-recover from the disease. As has already been explained the model is based on the SIR compartment model that has been explained in Figure 1 and
Figure 2. The NetLogo interface that shows the output of the environmental and disease parameters are shown in Figure 4 and Figure 5.

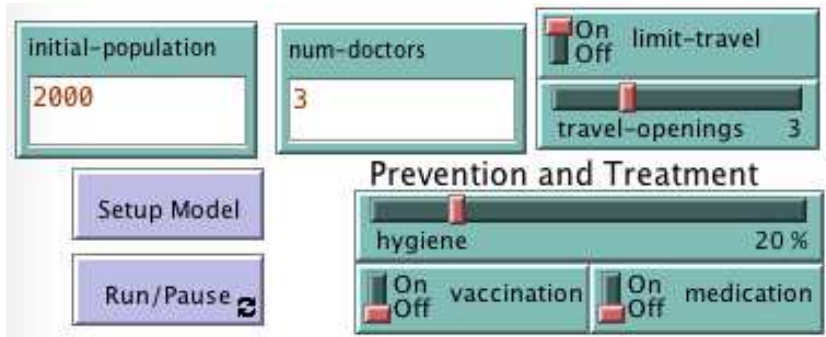

Figure 4. NetLogo interface showing environmental condition parameters.

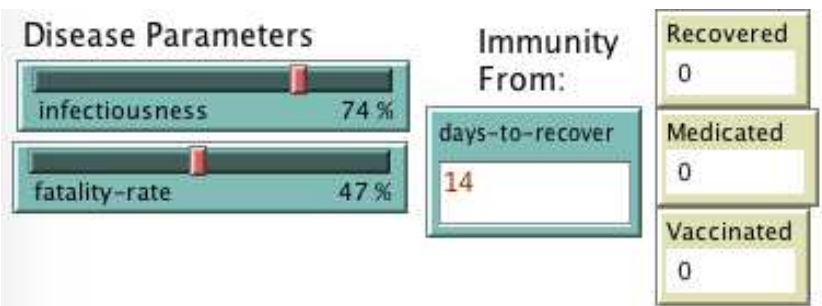

Figure 5. NetLogo interface showing disease parameters.

\subsection{Phase 3}

In this phase of the model, travel openings were included to make the model mimic inter-epidemic spread. It must be noted that those who come down with the disease seem not to be static and also there is random human movement which affects the spread and length of an epidemic. Figure 6 shows the NetLogo interface demonstrating the travel openings.

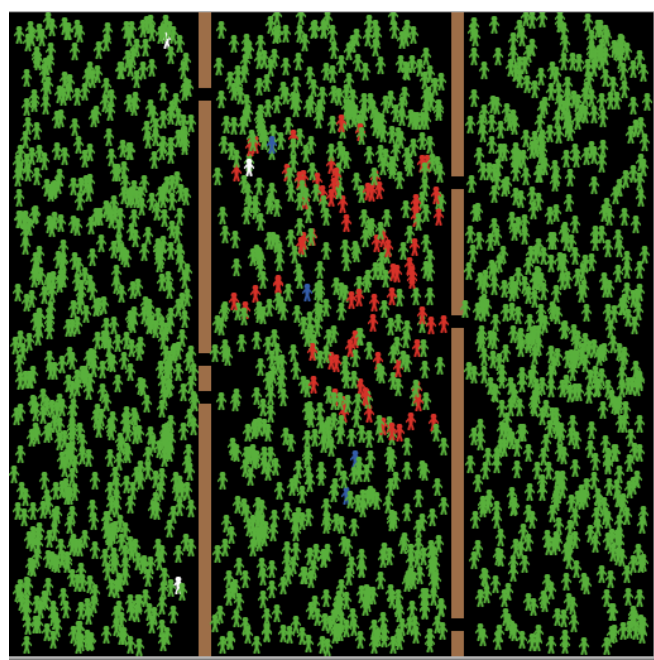

Figure 6. NetLogo interface showing the travel openings.

\section{Results and Discussion}

Information about the outbreak of Ebola in a community in Liberia from March 2014 to June 2014 was obtained from the Economist, a Graphic blog and then compared with values obtained from the model with the same parameters to test the performance of the model. Table 1 shows the comparison 
between the live and the simulated data. The information in Table 1 is graphically represented in Figure 7. It is obvious from the figure that the simulated results almost coincide with the results of the model. The model was further tested with results from the spread of influenza in a small town somewhere in Columbia by the Center for Disease Control and Prevention. The spread was studied for 42 weeks. The respective parameters were used to run a simulation with the model and the results were compared with that of the real-life data. Table 2 shows the comparison between the data obtained from the simulation and that from real-life situation for a population of 10,837 . Figure 8 also shows a graph of the comparison from the spread of the Influenza using Table 2.

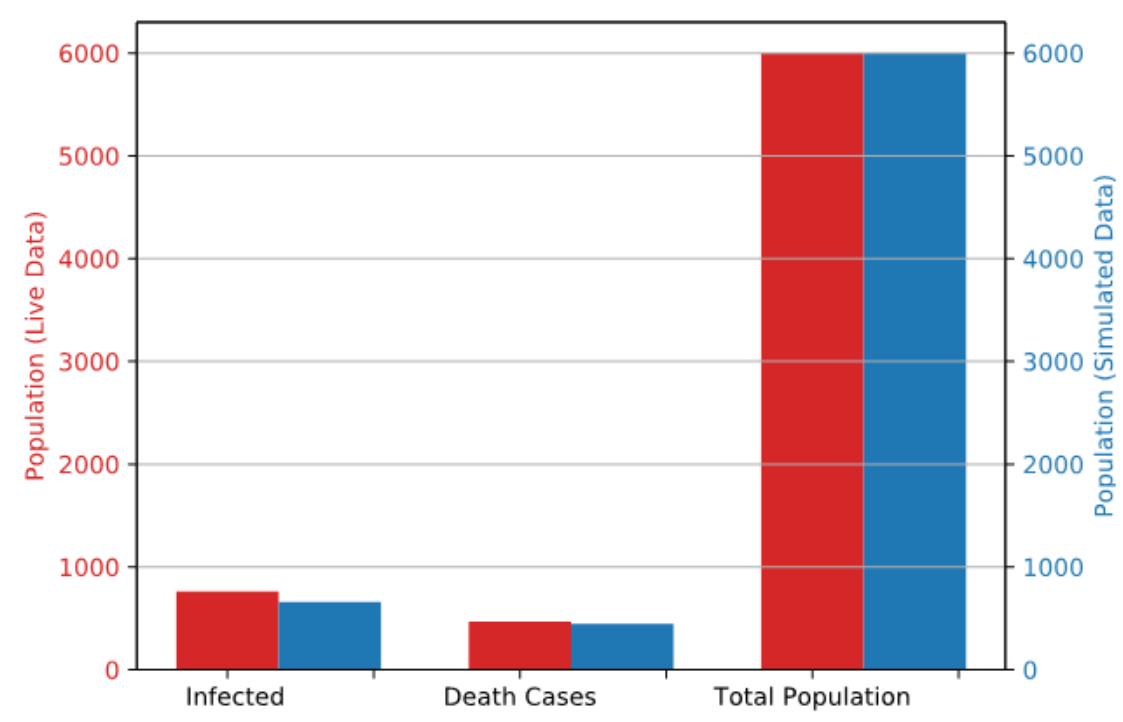

Figure 7. Graph showing the comparison of live and simulated data for Ebola.

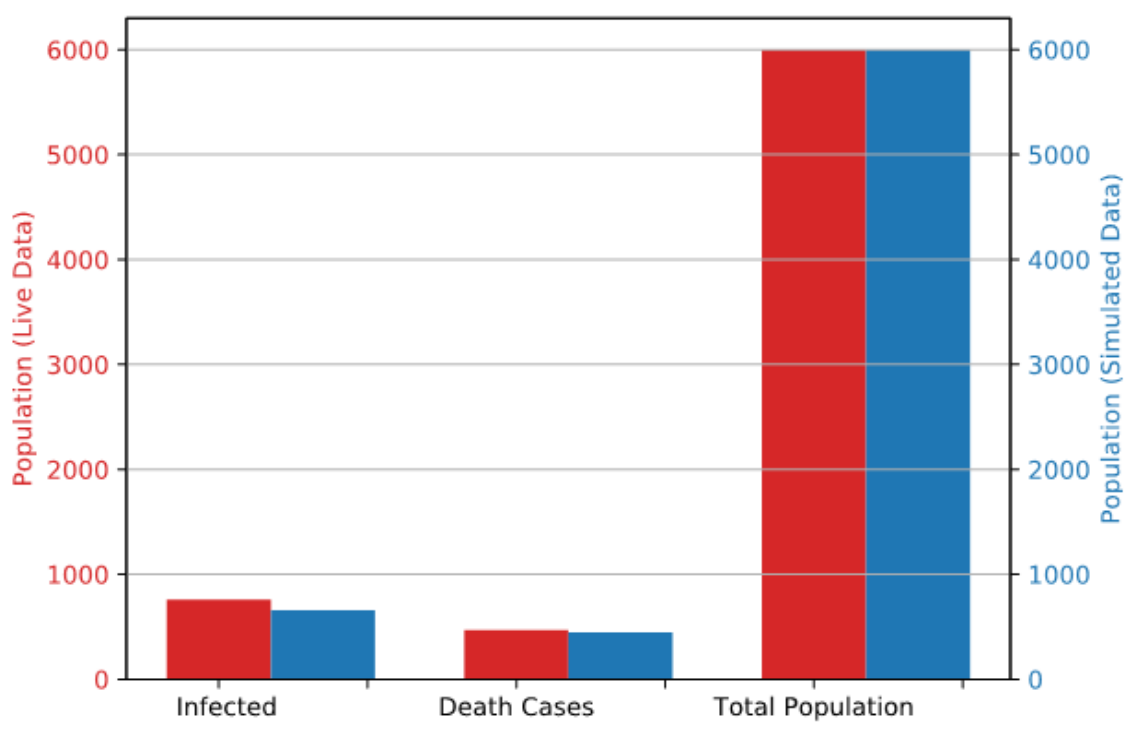

Figure 8. Graph showing the comparison of live and simulated data for Influenza.

Table 1. Comparison Between Live and Simulated Data for Ebola.

\begin{tabular}{lll}
\hline EBOLA & INFECTED & DEATH CASES \\
\hline LIVE DATA & 759 & 467 \\
SIMULATED DATA & 657 & 446 \\
\hline
\end{tabular}

Table 2. Comparison Between Live and Simulated Data for Ebola.

\begin{tabular}{lll}
\hline INFLUENZA & INFECTED & DEATH CASES \\
\hline LIVE DATA & 2605 & 2319 \\
SIMULATED DATA & 2811 & 2430 \\
\hline
\end{tabular}

\section{Conclusion and Recommendation}

This paper has presented a very simple model that can be used to predict the extent of an epidemic spread. From Figures 7 and 8 , the proposed model is quite close to that of the live data. This is mainly because, the movement of the turtles (humans) is random. It is recommended that effective quarantine measures should be taken in relation to diseases that produce a high infection and death rate. Future works 
will explore machine learning techniques that can be used to forecast the extent of an epidemic spread.

\section{Acknowledgements}

The authors acknowledge the College of Engineering, specifically, the department of Electrical and Electronic Engineering of the Kwame Nkrumah University of Science and Technology (KNUST), Kumasi-Ghana.

\section{References}

[1] Tom Britton, Etienne Pardoux, "Stochastic epidemics on homogenous community", arXiv: 1808.05350v1 [math.PR], pg. 5, August 2018.

[2] Z. Bai and Y. Zhou, "Existence of two periodic solutions for a non-autonomous SIR epidemic model”, Appl. Math. Model. 35 (2011), pp. 382-391.

[3] D. J. Earn, P. Rohani, B. M. Bolker, and B. T. Grenfell, A simple model for complex dynamical transitions in epidemics, Science 287 (2000), pp. 667-670.

[4] D. Greenhalgh and I. A. Moneim, SIRS epidemic model and simulations using different types of seasonal contact rate, Syst. Anal. Model. Simul. 43 (2003), pp. 573-600.

[5] J. Ma and Z. Ma, Epidemic threshold conditions for seasonally forced SEIR models, Math. Biosci. Eng. 3 (2006), pp. 161-172.

[6] C. Milling, C. Caramanis, S. Mannor, "Detecting epidemics using highly noisy data: Identifying the causative network of an epidemic", 2012.

[7] M. Hall, R. Gani, H. E. Hughes, S. Leach, "Real-time epidemic forecasting for pandemic influenza", Epidemol. Infect. (2007), 135, 372-385.
[8] Serfling RE, "Methods for current statistical analysis of excess pnuemonia-influenza deaths". Public Health Reports 1963; 78: 494-506.

[9] Costagliola D, et al. "A routine tool for detection and assessment of epidemics of influenza-like-illness", American Journal of Public Health 1991; 81: 97-99.

[10] Toubiana L, Flahault A. A space-time criterion for early detection of epidemics of influenza-like-illness. European Journal of Epidemiology 1998; 14: 465-470.

[11] Carrat F, Valleron AJ, "Epidemiologic mapping using the kriging method: application to an influenza-like illness epidemic in France". American Journal of Epidemiology 1992; 135: 1293-1300.

[12] Viboud C, et al. "Prediction of the spread of influenza epidemics by the method of analogues". American Journal of Epidemiology 2003; 158: 996-1006.

[13] Quenel P, Dab W. "Influenza A and B epidemic criteria based on time-series analysis of health services surveillance data". European Journal of Epidemiology 1998; 14: 275-285.

[14] Hashimoto S, et al. "Detection of epidemics in their early stage through infectious disease surveillance". International Journal of Epidemiology 2000; 29: 905-910.

[15] Mooney J, Wright E, Christie P. "Predictive modelling of influenza outbreaks: a linear regression analysis". SCIEH Weekly Report 2001; 35: 134-135.

[16] Mills CE, Robins JM, Lipsitch M. Transmissibility of 1918 pandemic influenza. Nature 2004; 432: 904-906.

[17] Wallinga J, Teunis P. Different epidemic curves for severe acute respiratory syndrome reveal similar impacts of control measures. American Journal of Epidemiology 2004; 160: 509-516. 